\title{
Comparison of High-Dose Dexamethasone and Prednisone for Initial Treatment of Adult Primary Immune Thrombocytopenia
}

\author{
Masanao Teramura, Midori Ishiyama, Hiroshi Kazama, Kentaro Yoshinaga, Masayuki Shiseki, \\ Naoki Mori, Toshiko Motoji
}

Department of Hematology, Tokyo Women's Medical University, Tokyo, Japan.

Email: teramura@dh.twmu.ac.jp

Received October $14^{\text {th }}, 2012$; revised November $18^{\text {th }}, 2012$; accepted November $30^{\text {th }}, 2012$

\begin{abstract}
Prednisone is the most common first-line treatment for adult primary immune thrombocytopenia (ITP). However, the best initial therapeutic approach is still a matter of debate. Prior studies have shown that high-dose dexamethasone (HD-DXM) produces a high sustained efficacy not achieved by conventional prednisone therapy. However, the definition of response widely differs between individual reports, and this heterogeneity makes comparison of the efficacy difficult. The aim of our study was to compare the therapeutic outcomes of a conventional dose of prednisone with HD-DXM for adult ITP patients as initial therapy. Thirty patients treated with prednisone and 22 patients treated HD-DXM were retrospectively analyzed. No significant differences between the HD-DXM and prednisone groups were observed for the rates of complete response (68\% vs. $70 \%)$ and response $(18 \%$ vs. $17 \%)$. However, 1 year probability of sustained response was significantly greater in the HD-DXM group than in the prednisone group (78\% vs. $38 \%$; $\mathrm{P}=$ 0.008). No adverse events necessitating discontinuation of treatment were observed in either group. Our retrospective analysis showed that initial treatment with HD-DXM produced longer response duration compared to a conventional dose of prednisone. Randomized clinical trials are warranted to establish the optimal initial steroid therapy for adult ITP.
\end{abstract}

Keywords: Primary Immune Thrombocytopenia; High-Dose Dexamethasone; Prednisone

\section{Introduction}

Primary immune thrombocytopenia (ITP) previously called idiopathic thrombocytopenic purpura is an acquired immune-mediated disorder characterized by isolated thrombocytopenia defined as a peripheral blood platelet count less than $100 \times 10^{9} / \mathrm{L}$, and the absence of any obvious initiating and/or underlying cause of the thrombocytopenia [1]. Treatment is generally given for patients with a low platelet count $\left(\leq 20 \times 10^{9} / \mathrm{L}\right.$ or $\leq 30 \times$ $10^{9} / \mathrm{L}$ ) or clinically significant bleeding. The standard initial treatment for adult patients with ITP is corticosteroids. Among corticosteroids, prednisone is widely regarded as the standard first-line treatment. The starting dose is usually set at $1 \mathrm{mg} / \mathrm{kg}$ per day and continued for 2 to 4 weeks. If an increase in platelet count is obtained, the dose is gradually tapered. With this approach, an initial response rate of $50 \%$ to $60 \%$ is obtained, although long-term remission rate after discontinuation are very low (10\% to $25 \%)$ [2-7].

A short course of high-dose dexamethasone (HD-DXM) has been used in patients with refractory ITP, but its efficacy is controversial [8-10]. In 2003, a study on initial treatment of ITP with HD-DXM found beneficial outcomes. In adult patients with previously untreated ITP, dexamethasone was given in a single 4-day course (40 $\mathrm{mg} /$ day, orally). The initial response rate was $85 \%$ of patients (106 of 125 patients), and the sustained response (a platelet count $>50,000 / \mu \mathrm{L} 6$ months after initial treatment) was $50 \%$ [11]. Subsequently, a multicenter study reported that $89 \%$ (16 of 18) ITP patients responded to initial HD-DXM treatment (1 to 6 courses) with 59\% achieving 2 - 31 months efficacy [12]. In another large cohort study, 4 cycles of HD-DXM given every 14 days for previously untreated ITP patients produced an $85.6 \%$ (77 of 90 patients) response rate with $74 \%$ efficacy lasting for a median duration of 8 months [13].

The results of these studies suggest that HD-DXM treatment for adult ITP may produce better outcomes than conventional prednisone therapy. However, the definition of response widely differs between individual 
reports, and this heterogeneity makes comparison of the efficacy of these two treatments difficult. To address this issue, in 2009, an international working group proposed standard criteria for assessing response to ITP treatments [1]. The aim of this study is to compare the efficacy of HD-DXM and conventional prednisone treatments using these criteria through the retrospective analysis of ITP patients treated at our institution.

\section{Patients and Method}

\subsection{Patient Selection}

Adult patients diagnosed with ITP and initially treated with either a conventional dose of prednisone or HDDXM between 1995-2011 at our institution were retrospectively analyzed for this study. Diagnosis of ITP was based on findings from complete blood cytology, and other laboratory testing, which were used to rule out other causes of thrombocytopenia. To confirm the diagnosis, bone marrow aspirate was obtained from all patients, and only those with the presence of a normal or increased number of megakaryocytes without pathologic alterations of erythroblasts, granulocytes or lymphocytes were in- cluded. In addition, all patients had either a platelet count $\leq 20 \times 10^{9} / \mathrm{L}$ or clinically significant bleeding with a platelet count $\leq 50 \times 10^{9} / \mathrm{L}$.

\subsection{Treatment}

For the HD-DXM group, oral dexamethasone was given as at least 1 cycle of a single daily dose of $40 \mathrm{mg}$ for 4 consecutive days. A maximum of 3 cycles of HD-DEX with a treatment interval of 14 days were given. For the prednisone group, $1 \mathrm{mg} / \mathrm{kg}$ per day of prednisone was given for 4 weeks, and in responders, the dose was gradually tapered over several weeks and then stopped. During the initial treatment, all patients did not receive other ITP-specific concomitant treatment including Helicobacter pylori eradication.

Response was evaluated according to the proposed criteria from an international working group [1]. Complete response (CR) was defined as any platelet count of at least $100 \times 10^{9} / \mathrm{L}$, and response $(\mathrm{R})$ was defined as any platelet count between 30 and $100 \times 10^{9} / \mathrm{L}$ and at least doubling of the baseline count. No response (NR) was defined as any platelet count lower than $30 \times 10^{9} / \mathrm{L}$ or less than doubling of the baseline count. The definition of response required concurrent resolution of bleeding symptoms. Loss of CR was defined as a CR patient whose platelet count later fell below $100 \times 10^{9} / \mathrm{L}$, or experienced bleeding. Loss of $\mathrm{R}$ was defined as a patient whose platelet count fell later below $30 \times 10^{9} / \mathrm{L}$, or to less than 2-fold increase of baseline platelet count or experienced bleeding. Patients who did not respond until 4 weeks after initiation of prednisone, and those who did not response until 2 weeks after completion of HD-DXM were defined as non-responders.

The duration of response was measured from the time of achievement of CR or R to time of loss of CR or R (from R). We also measured the duration of response from the time of achievement of CR or R to loss of $\mathrm{R}$ (from CR or R). This study was approved by the ethical committee of Tokyo Women's Medical University.

\subsection{Statistical Analysis}

Prednisone and HD-DXM groups were compared with respect to age, sex, and platelet counts before treatment, initial response rate, and the duration of response. Fisher's exact test was used to compare categorical variables, and Mann-Whitney $U$ test or Student's $t$-test was used to compare continuous variables. The duration of response was analyzed using the Kaplan-Meier method [14].

\section{Results}

\subsection{Patient Characteristics}

Fifty two patients diagnosed of ITP were analyzed, of whom 30 were treated with prednisone and 22 treated with HD-DXM. The main clinical characteristics of both groups are listed in Table 1. There were no significant differences between the 2 groups in terms of age, sex, and the pretreatment white blood cell count, platelet count, and Hemoglobin level. In the HD-DXM group, 11, 4 , and 7 patients received 1,2 , or 3 treatment cycles, respectively.

\subsection{Treatment Response}

The rates of CR, $\mathrm{R}$ and overall response (CR plus $\mathrm{R}$ ) are shown in Table 2. No significant differences between the two treatments groups were observed with respect to the rate of $\mathrm{CR}, \mathrm{R}$, and overall response.

\subsection{Duration of Response}

Loss of CR and loss of R (from R) were observed in 10 of 19 patients in the HD-DXM group with a median time of 5.8 months (range 1 - 71 months) after response achievement, and in 22 of 26 patients in the prednisone group with a median time of 5.9 months (range 1 - 147 months). Loss of R (from CR or R) was observed in 5 of 19 patients in the HD-DXM group with a median time of 13.6 months (range 1 - 75 months) after response achievement, and in 20 of 26 patients in the prednisone group with a median duration of 8.4 months (range 1 - 147 months).

Kaplan-Meier response duration curves for 2 groups were shown in Figure 1. Response duration calculated 
Table 1. Clinical characteristics of patients treated with high-dose dexamethasone and prednisone.

\begin{tabular}{cccc}
\hline & HD-DXM & Prednisone & P value \\
\hline No. of patients & 22 & 30 & \\
Age (year) & $66(23-84)^{*}$ & $56(20-73)^{*}$ & 0.06 \\
Sex (male/female) & $6 / 16$ & $10 / 20$ & 0.76 \\
WBC $\left(\times 10^{9} / \mathrm{L}\right)$ & 5.3 & 5.4 & 0.29 \\
& $(2.6-8.7)^{*}$ & $(3.7-9.3)^{*}$ & \\
Hb (g/dL) & 13.0 & 13.3 & 0.39 \\
& $(7.2-16.1)^{*}$ & $(10.2-15.7)^{*}$ & \\
Platelet $\left(\times 10^{9} / \mathrm{L}\right)$ & 12 & 13 & 0.27 \\
& $(2-32)^{*}$ & $(2-50)^{*}$ & \\
\hline
\end{tabular}

*Median (range); HD-DXM: high-dose dexamethasone; WBC: white blood cell count; Hb: Hemoglobin.

Table 2. Patient outcomes.

\begin{tabular}{cccc}
\hline & HD-DXM & Prednisone & \\
\cline { 2 - 3 } & No. (\%) & No. (\%) & \\
\hline Complete response (CR) & $15(68)$ & $21(70)$ & 0.562 \\
Response (R) & $4(18)$ & $5(17)$ & 0.585 \\
Overall response (CR + R) & $19(86)$ & $26(87)$ & 0.641 \\
No response (NR) & $3(14)$ & $4(13)$ & 0.641 \\
\hline
\end{tabular}

HD-DXM: high-dose dexamethasone; Complete response (CR) was defined as any platelet count of at least $100 \times 10^{9} / \mathrm{L}$; and response $(\mathrm{R})$ was defined as any platelet count between 30 and $100 \times 10^{9} / \mathrm{L}$ and at least doubling of the baseline count; No response (NR) was defined as any platelet count lower than $30 \times 10^{9} / \mathrm{L}$ or less than doubling of the baseline count.

from the time of CR or R until loss of CR or R (from R) was not statistically different between 2 groups (1 year probability of response duration: HD-DXM group; $56 \%$, prednisone group; 23\%, P = 0.09) (Figure 1(a)). Response duration calculated from the time of CR or R until loss of R was statistically different between 2 groups (1 year probability of response duration: HD-DXM group; $78 \%$, prednisone group; $38 \%, \mathrm{P}=0.008)$ (Figure 1(b)).

\subsection{Adverse Events}

No adverse events necessitating the discontinuation of treatment were observed in either group. Steroid induced hyperglycemia was observed in 2 patients in the HDDXM group and in 3 patients in the prednisone group. In the prednisone group, 1 patient experience vertebral bone compression fracture, and a transient increase in pancreatic enzyme was observed in 1 patient in each group.
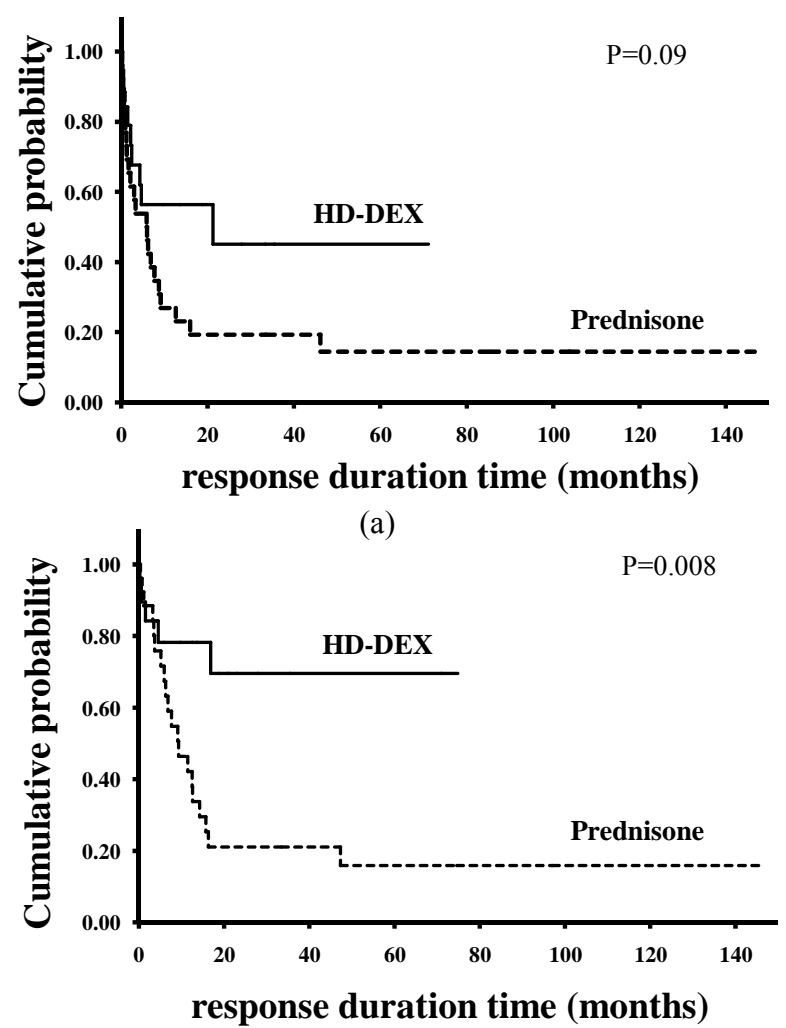

(b)

Figure 1. Kaplan-Meier response duration curves. (a) Response duration from the time of $C R$ or $R$ until loss of $C R$ or $R$ (from R); (b) Response duration from the time of $C R$ or $R$ until loss of $R$. HD-DXM group versus prednisone group: (a) $P=0.09$; (b) $P=0.008$. Solid line: HD-DXM, Broken line: Prednisone.

\section{Discussion}

We found HD-DXM and prednisone to have nearly identical initial response rates for the treatment of ITP (CR: $68 \%$ vs. $70 \%$, and R: $18 \%$ vs. $17 \%$ ). However, 1 year probability of response duration calculated from the time of CR or R until loss of R was significantly greater in the HD-DXM group than in the prednisone group $(78 \%$ vs. $38 \%$ ). Our results therefore support the previous studies that found HD-DXM to produce long-term responses in previously untreated ITP patients [11-13]. In a study by Cheng et al., a single course of HD-DXM for adult ITP produced a $50 \%$ sustained response of platelet count $\geq$ $50,000 / \mu \mathrm{L}$ at 6 months after the initial treatment [11]. A multicenter study by Borst et al. found that $59 \%$ of previously untreated adult ITP patients obtained a sustained response 2 - 31 months after 1 - 6 cycles of HD-DXM therapy [12]. A large cohort study by Mazzucconi et al. found that 4 cycles of HD-DXM given every 14 days in previously untreated adult ITP patients achieved a relapse-free response rate of $60 \%$ at 15 months [13]. 
The optimal number of cycles of HD-DXM for initial ITP treatment warrants further investigation. Cheng et al. [11] used a single course of HD-DXM, while Borst et al. [12], Mazzucconi et al. [13], and we used between 1 - 6 courses. In the study from Mazzucconi et al., response rate was significantly improved between the second and third cycles $(75.8 \%$ vs. $89 \%, \mathrm{P}=0.018)$ but not between the third and fourth [13]. This suggests that 3 cycles of HD-DXM may be appropriate.

The mechanism of action of HD-DXM in the treatment of ITP is not entirely clear; however, several mechanisms have been postulated. ITP is an autoimmune disease characterized by $\mathrm{T}$ helper 1 (Th1) polarization. Guo et al. reported that correction of Th1 polarization was achieved and maintained after HD-DXM therapy in patients who experienced a sustainable response but not maintained in those who relapsed [15]. A subsequent study from the same group showed that HD-DXM reduced plasma levels of interleukin-18 (IL-18) while increasing levels of its endogenous antagonist, IL-18 binding protein (IL-18BP), yielding a reduction of IL-18/IL18BP ratio [16]. From these findings, it is suggested that correction of Th1 polarization by HD-DXM is mediated by a decrease in IL-18/IL-18BP ratio, and this could be a potential mechanism of the long-term recovery from ITP.

Disruptions in the balance of $\mathrm{Fc} \gamma$ receptors ( $\mathrm{F} c \mathrm{Rs}$ ), including Fc $\gamma$ RI, Fc $\gamma$ RIIa, Fc $\gamma$ RIII, and Fc $\gamma$ RIIb have been implicated in the pathogenesis of many autoimmune diseases. In ITP, decreased expression of inhibitory Fc $\gamma$ RIIb and elevated expression of activating Fc $\gamma$ Rs (Fc $\gamma$ RIIa and Fc $\gamma$ RIII) have been observed on monocytes, and HDDXM therapy for ITP could shift monocyte Fc $\gamma \mathrm{R}$ balance toward the inhibitory $\mathrm{Fc} \gamma \mathrm{R} \mathrm{IIb}$, resulting in the decrease of monocyte phagocytic capacity [17]. This suggests that $\mathrm{F} c \gamma \mathrm{R}$ system is possibly involved in the efficacy of HD-DXM for ITP. Other reports have suggested that HD-DXM may achieve its therapeutic effect by inhibiting immune responses through the suppression of dendritic cell functions [18], reducing B-cell activating factor production [19], and increasing production of the regulatory T-cells [20].

Recently, a prospective randomized study involving previously untreated adult ITP comparing the efficacy of a single course of HD-DEX to that of HD-DEX combined with rituximab was conducted [21]. The rate of sustained response (platelet count $>50 \times 10^{9} / \mathrm{L}$ at 6 months after treatment) was significantly higher in patients treated with HD-DEX plus rituximab than in those treated with HD-DXM alone (63\% vs. $36 \%)$. Furthermore, the HD-DXM plus rituximab regimen was effecttive as salvage therapy in the subgroup of patients who were refractory to initial HD-DXM therapy, achieving a sustained response rate of $56 \%$. This suggests that com- bining HD-DEX with rituximab may be a promising second-line therapy in patients not responding to initial HD-DEX treatment.

There are several limitations to our study. First, because our study is retrospective in nature, some bias may have been present. The dates of treatment tended to be different between HD-DEX and prednisone groups; all HD-DEX patients were treated after 2003, whereas most prednisone patients were treated before 2003. In addition, in Japan, Helicobacter pylori eradication was adopted as initial therapy for ITP patients during 2000s [22]. Therefore, another limitation is that the extent of $\mathrm{H}$. pylori infection in the 2 groups was likely different, and the possibility that this etiological difference affected our findings cannot be ruled out.

In conclusion, treatment involving steroid therapy for ITP is used to obtain not only a high initial response rate but also a sustainable response that avoids the need for any further treatment. Based on our findings, HD-DXM could be used as a first-line treatment for adult patients with ITP because it accomplishes both of these. Our retrospective analysis showed that initial treatment with HD-DXM produced longer response duration compared to a conventional dose of prednisone. Randomized clinical trials are warranted to establish the optimal initial steroid therapy for adult ITP.

\section{Acknowledgements}

We thank all members of the Department of Hematology, Tokyo Women's Medical University for their support. We also thank Editage for providing editorial assistance.

\section{REFERENCES}

[1] F. Rodeghiero, R. Stasi, T. Gernsheimer, M. Michel, D. Provan, D. M. Arnold, et al., "Standardization of Terminology, Definitions and Outcome Criteria in Immune Thrombocytopenic Purpura of Adults and Children: Report from an International Working Group," Blood, Vol. 113, No. 11, 2009, pp. 2386-2393. doi:10.1182/blood-2008-07-162503

[2] S. Bellucci, Y. Charpak, C. Chastang and G. Tobelem, "Low Doses V Conventional Doses of Corticoids in Immune Thrombocytopenic Purpura (ITP): Results of a Randomized Clinical Trial in 160 Children, 223 Adults," Blood, Vol. 71, No. 4, 1988, pp. 1165-1169.

[3] D. B. Cines and V. S. Blanchette, "Immune Thrombocytopenic Purpura," New England Journal of Medicine, Vol. 346, No. 13, 2002, pp. 995-1008. doi:10.1056/NEJMra010501

[4] J. N. George, M. A. EL-Harake and G. E. Raskob, "Chronic Idiopathic Thrombocytopenic Purpura," New England Journal of Medicine, Vol. 331, No. 18, 1994, pp. 1207-1211. doi:10.1056/NEJM199411033311807 
[5] J. N. George, S. H. Woolf, G. E. Raskob, J. S. Wasser, L. M. Aledort, P. J. Ballem, et al., "Idiopathic Thrombocytopenic Purpura: A Practice Guideline Developed by Explicit Methods for the American Society of Hematology," Blood, Vol. 88, No. 1, 1996, pp. 3-40.

[6] M. G. Mazzucconi, M. Francesconi, P. Fidani, G. Di Nucci, G. M. Gandolfo, A. Afeltra, et al., "Treatment of Idiopathic Thrombocytopenic Purpura (ITP): Results of a Multicentric Protocol," Haematologica, Vol. 70, No. 4, 1985, pp. 329-336.

[7] J. E. Portielje, R. G. Westendorp, H. C. Kluin-Nelemans and A. Brand, "Morbidity and Mortality in Adults with Idiopathic Thrombocytopenic Purpura," Blood, Vol. 97, No. 9, 2001, pp. 2549-2554. doi:10.1182/blood.V97.9.2549

[8] J. C. Andersen, "Response of Resistant Idiopathic Thrombocytopenic Purpura to Pulsed High-Dose Dexamethasone Therapy," New England Journal of Medicine, Vol. 330, No. 22, 1994, pp. 1560-1564. doi:10.1056/NEJM199406023302203

[9] I. Khouri, B. Tuan and K. Grant, "Comment on: Immune Thrombocytopenic Purpura," New England Journal of Medicine, Vol. 347, No. 6, 2002, pp. 449-450. doi:10.1056/NEJM200208083470617

[10] M. Warner, P. Wasi, S. Couban, C. Hayward, T. Warkentin and J. D. Kelton, "Failure of Pulse High-Dose Dexamethasone in Chronic Idiopathic Immune Thrombocytopenia," American Journal of Hematology, Vol. 54, No. 4, 1997, pp. 267-270. doi:10.1002/(SICI)1096-8652(199704)54:4<267::AID-AJ H1>3.0.CO;2-T

[11] Y. Cheng, R. S. Wong, Y. O. Soo, C. H. Chui, F. Y. Lau, N. P. Chan, et al., "Initial Treatment of Immune Thrombocytopenic Purpura with High-Dose Dexamethasone," New England Journal of Medicine, Vol. 349, No. 9, 2003, pp. 831-836. doi:10.1056/NEJMoa030254

[12] F. Borst, J. J. Keuning, H. van Hulsteijn, H. Sinnige and G. Vreugdenhil, "High-Dose dexamethasone as a Firstand Second-Line Treatment of Idiopathic Thrombocytopenic Purpura in Adults," Annals of Hematology, Vol. 83, No. 12, 2004, pp. 764-768. doi:10.1007/s00277-004-0908-1

[13] M. G. Mazzucconi, P. Fazi, S. Bernasconi, G. De Rossi, G. Leone, L. Gugliotta, et al., "Therapy with High-Dose Dexamethasone (HD-DXM) in Previously Untreated Patients Affected by Idiopathic Thrombocytopenic Purpura: A GIMEMA Experience," Blood, Vol. 109, No. 4, 2007, pp. 1401-1407. doi:10.1182/blood-2005-12-015222

[14] E. L. Kaplan and P. Meier, "Nonparametric Estimation from Incomplete Observations," Journal of the American
Statistical Association, Vol. 53, No. 282, 1958, pp. $457-$ 481. doi: $10.2307 / 2281868$

[15] C. Guo, X. Chu, Y. Shi, W. He, L. Li, L. Wang, et al., "Correction of Th1-Dominant Cytokine Profiles by HighDose Dexamethasone in Patients with Chronic Idiopathic Thrombocytopenic Purpura," Journal of Clinical Immunology, Vol. 27, No. 6, 2007, pp. 557-562. doi:10.1007/s10875-007-9111-1

[16] N. N. Shan, X. J. Zhu, Q. Wang, C. Y. Wang, P. Qin, J. Peng, et al., "High-Dose Dexamethasone Regulates Interleukin-18 and Interleukin-18 Binding Protein in Idiopathic Thrombocytopenic Purpura," Haematologica, Vol. 94, No. 11, pp. 1603-1607. doi:10.3324/haematol.2009.007708

[17] X. G. Liu, S. H. Ma, J. Z. Sun, J. Ren, Y. Shi, L. Sun, et al., "High-Dose Dexamethasone Shifts the Balance of Stimulatory and Inhibitory Fcgamma Receptors on Monocytes in Patients with Primary Immune Thrombocytopenia," Blood, Vol. 117, No. 6, 2011, pp. 2061-2069. doi:10.1182/blood-2010-07-295477

[18] X. X. Chu, B. H. Huang, X. L. Zhang, L. M. Chen, Y. Wang, W. L. Yu, et al., "Dexamethasone Inhibits Immunoreactivity of Dendritic Cells in Patients with Chronic Idiopathic Thrombocytopenic Purpura," Blood Coagulation \& Fibrinolysis, Vol. 21, No. 6, 2010, pp. 564-567. doi:10.1097/MBC.0b013e32833c2b8c

[19] X. J. Zhu, Y. Shi, J. Z. Sun, N. N. Shan, J. Peng, C. S. Guo, et al., "High-Dose Dexamethasone Inhibits BAFF Expression in Patients with Immune Thrombocytopenia," Journal of Clinical Immunology, Vol. 29, No. 5, 2009, pp. 603-610. doi:10.1007/s10875-009-9303-y

[20] Y. Ling, X. Cao, Z. Yu and C. Ruan, "Circulating Dendritic Cells Subsets and CD4+ Foxp3+ Regulatory TCells in Adult Patients with Chronic ITP before and after Treatment with High-Dose Dexamethasome," European Journal of Haematology, Vol. 79, No. 4, 2007, pp. 310316. doi:10.1111/j.1600-0609.2007.00917.x

[21] F. Zaja, M. Baccarani, P. Mazza, M. Bocchia, L. Gugliotta, A. Zaccaria et al., "Dexamethasone plus Rituximab Yields Higher Sustained Response Rates than Dexamethasone Monotherapy in Adults with Primary Immune Thrombocytopenia," Blood, Vol. 115, No. 14, 2010, pp. 2755-2762. doi:10.1182/blood-2009-07-229815

[22] K. Fujimura, M. Kuwana, Y. Kurata, M. Imamura, H. Harada, H. Sakamaki, et al., "Is Eradication Therapy Useful as the First Line of Treatment in Helicobacter Pylori-Positive Idiopathic Thrombocytopenic Purpura? Analysis of 207 Eradicated Chronic ITP Cases in Japan," International Journal of Hematology, Vol. 81, No. 2, 2005, pp. 162-168. doi:10.1532/IJH97.04 